\title{
A novel approach to perioperative risk assessment for patients with pulmonary hypertension
}

\author{
Hussein J. Hassan ${ }^{1}$, Traci Housten ${ }^{1}$, Aparna Balasubramanian ${ }^{1}$, Catherine E. Simpson $\mathbb{D}^{1}$, \\ Rachel L. Damico ${ }^{1}$, Stephen C. Mathai ${ }^{1}$, Paul M. Hassoun ${ }^{1}$, Jochen Steppan ${ }^{2}$, Peter J. Leary ${ }^{3}$ and \\ Todd M. Kolb $\mathbb{B D}^{1}$
}

${ }^{1}$ Dept of Medicine, Division of Pulmonary and Critical Care Medicine, Johns Hopkins University, Baltimore, MD, USA. ${ }^{2}$ Dept of Anesthesiology and Critical Care Medicine, Johns Hopkins University, Baltimore, MD, USA. ${ }^{3}$ Dept of Medicine, Division of Pulmonary, Critical Care and Sleep Medicine, University of Washington, Seattle, WA, USA.

Corresponding author: Todd M. Kolb (toddkolb@jhmi.edu)

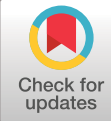

This version is distributed under the terms of the Creative Commons Attribution NonCommercial Licence 4.0. For commercial reproduction rights and permissions contact permissions@ersnet.org

This article has supplementary material available from openres.ersjournals.com

Received: 12 April 2021 Accepted: 2 May 2021

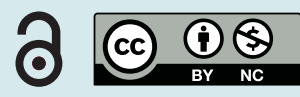

\begin{abstract}
Shareable abstract (@ERSpublications)
For patients with pulmonary hypertension undergoing noncardiac surgery, perioperative risk can be estimated using a model that combines inherent procedural risk with composite PAH risk assessment scores https://bit.ly/3uTCibP
\end{abstract}

Cite this article as: Hassan HJ, Housten T, Balasubramanian A, et al. A novel approach to perioperative risk assessment for patients with pulmonary hypertension. ERJ Open Res 2021; 7: 002572021 [DOI: 10.1183/23120541.00257-2021].

\section{Abstract}

Rationale Pulmonary hypertension (PH) is associated with significant perioperative morbidity and mortality. We hypothesised that pulmonary arterial hypertension (PAH) composite risk assessment scores could estimate perioperative risk for $\mathrm{PH}$ patients when adjusted for inherent procedural risk.

Methods We identified patients in the Johns Hopkins PH Center Registry that had noncardiac surgery (including endoscopies) between September 2015 and January 2020. We collected information on preoperative patient-level and procedural variables and used logistic regression to evaluate associations with a composite outcome of death within 30 days or serious postoperative complication. We generated composite patient-level risk assessment scores for each subject and used logistic regression to estimate the association with adverse surgical outcomes. We adjusted multivariable models for inherent procedural risk of major cardiovascular events and used these models to generate a numerical $\mathrm{PH}$ perioperative risk (PHPR) score.

Results Among 150 subjects, 19 (12.7\%) reached the primary outcome, including 7 deaths (4.7\%). Individual patient-level and procedural variables were associated with the primary outcome (all $\mathrm{p}<0.05$ ). A composite patient-level risk assessment score built on three noninvasive parameters was strongly associated with reduced risk for poor outcomes $(O R=0.4, p=0.03)$. This association was strengthened after adjusting the model for procedural risk. A PHPR score derived from the multivariable model stratified patients into low $(0 \%)$, intermediate $(\leqslant 10 \%)$, or high $(>10 \%)$ risk of reaching the primary outcome.

Conclusion Composite PAH risk assessment scores can predict perioperative risk for $\mathrm{PH}$ patients after accounting for inherent procedural risk. Validation of the PHPR score in a multicentre, prospective cohort is warranted.

\section{Introduction}

Perioperative risk assessment presents a formidable challenge for providers managing patients with pulmonary hypertension (PH). Multiple studies have demonstrated increased risk of mortality and serious morbidity among PH patients undergoing noncardiac surgery [1-6], and some have identified $\mathrm{PH}$ as an independent risk for perioperative complications and death [6-8]. This clinical challenge becomes increasingly relevant for patients with pulmonary arterial hypertension (PAH) as advances in medical therapy expand the population of patients requiring invasive procedures [9]. Current PH guidelines [10, 11] provide limited guidance for assessing perioperative risk. Better risk assessment tools may assist providers and patients in complex perioperative decision-making. 
Previous studies have identified preoperative variables associated with poor surgical outcomes for patients with $\mathrm{PH}[1,3,5,8]$. The clinical utility of these associations has been limited, as they have been inconsistent among studies with differing patient populations, surgical procedures, and small samples sizes. In parallel, there is a growing effort to address longitudinal clinical risk assessment for PAH patients, unrelated to surgery. Several approaches have been used to combine multiple clinical variables into composite risk assessment scores [11-16]. These scoring systems have been validated in predicting clinical [12-14, 17, 18] and patient-centred [19] outcomes in diverse PAH groups. We hypothesised that composite risk assessment scores could estimate perioperative risk for $\mathrm{PH}$ patients when adjusted for procedural risk. Moreover, we present "proof-of-concept" data showing that this approach can be used to develop a clinically pragmatic perioperative risk assessment tool.

Methods

Study population and data extraction

We included participants in the Johns Hopkins Pulmonary Hypertension Center Registry (Institutional Review Board no. NA_00027124), which enrolls consecutive patients from all World Symposium on Pulmonary Hypertension (WSPH) groups [20]. From September 2015 to January 2020, we identified upcoming surgical procedures among Registry participants during weekly meetings, including all noncardiac surgical procedures (excluding lung transplantation) requiring at least moderate sedation. Some patients had multiple procedures during the study period; only the last procedure was analysed.

\section{Preoperative patient-level risk variables of interest}

Individual patient-level risk variables were selected a priori from previous studies evaluating operative risk in $\mathrm{PH}[1-4,7,8]$ and studies evaluating predictors of overall survival in PAH [11, 13-16, 21, 22]. These included American Society of Anesthesiologists' (ASA) classification, World Health Organization functional class (WHO FC), serum brain natriuretic peptide (BNP) or N-terminal pro-hormone BNP (NT-proBNP) level, 6-min walking distance (6MWD), echocardiographic parameters (right atrial and ventricular dilation, tricuspid annular plane systolic excursion (TAPSE), maximum Doppler tricuspid regurgitant velocity), and right heart catheterisation parameters (right atrial pressure (RAP), cardiac index, mixed venous oxygen saturation $\left(S_{\mathrm{vO}_{2}}\right)$ ). We used multiple approaches to generate composite risk assessment scores [12-14] from patient-level risk variables as defined in current PAH guidelines [11]. In one approach ("low-risk focused”), we quantified the number of "low-risk" features present among three noninvasive parameters (WHO FC I or II, 6MWD $>440 \mathrm{~m}$, BNP $<50 \mathrm{ng} \cdot \mathrm{L}^{-1}$ or NT-proBNP $<300 \mathrm{ng} \cdot \mathrm{L}^{-1}$ ) or a combination of four invasive and noninvasive parameters (WHO FC I or II, 6MWD $>440 \mathrm{~m}$, RAP $<8 \mathrm{mmHg}$, cardiac index $\geqslant 2.5 \mathrm{~L} \cdot \mathrm{min}^{-1} \cdot \mathrm{m}^{-2}$ ) to generate composite scores [14]. In another approach ("score and average"), we assigned a value of 1 (low risk), 2 (intermediate risk), or 3 (high risk) for each of six clinical parameters (supplemental table S1), then averaged the values to generate a composite score [13]. In all cases, we used the closest measurement before the surgical procedure for analyses or composite risk assessment score calculation.

\section{Procedural risk variables of interest}

We identified procedural risk variables of interest using American College of Cardiology/American Heart Association (ACC/AHA) perioperative guidelines [23] and previous studies [3, 4, 7, 8, 24]. These included inherent risk of major cardiovascular events ("elevated" or $\geqslant 1 \%$ risk versus "low" or $<1 \%$ risk), emergency procedure (patient requires access to the operating room within $24 \mathrm{~h}$ of the decision to operate [25]), length of procedure $(>3 \mathrm{~h}$ ), and location of patient immediately prior to procedure (inpatient versus outpatient).

\section{Outcome of interest}

The primary outcome was the composite of death within 30 days or serious postoperative complication. Serious postoperative complications required intensive care unit (ICU) admission and included any of the following: 1) haemodynamic instability requiring inotropes and/or vasopressors; 2) respiratory failure requiring invasive mechanical ventilation $\geqslant 48 \mathrm{~h}$; 3) initiation of inhaled pulmonary vasodilators; 4) acute coronary syndrome; 5) cerebrovascular accident; 6) arrhythmia; 7) renal failure (serum creatinine $>2 \mathrm{mg} \cdot \mathrm{dL}^{-1}$ for patients with normal preoperative levels, or a $50 \%$ increase in serum creatinine, or initiation of dialysis); 8 ) hepatic injury (elevation of aspartate aminotransferase and/or alanine aminotransferase by $50 \%$ or more); and 9) sepsis [1-3, 8, 26]. Secondary outcomes included postoperative hospital length of stay (LOS), ICU LOS, and hospital readmission for a medical deterioration within 30 days of the procedure.

\section{Statistical analysis}

For descriptive analyses, we used the independent t-test for normally distributed continuous variables, the Wilcoxon rank-sum test for skewed continuous variables, and the Chi-squared test for categorical variables. We used univariable logistic regression to evaluate associations between preoperative variables and the 
primary outcome. For secondary outcomes, we used linear regression to estimate associations between preoperative variables and hospital or ICU LOS and logistic regression to estimate the association with readmission within 30 days.

We used logistic regression to estimate associations between composite risk assessment scores ("low-risk focused" and "score and average") and the primary outcome. We used multivariable regression to account for differences in procedural risk based on inherent risk of major cardiovascular events (elevated versus low) [23]. We selected this variable to capture procedural risk because it had the strongest association with the primary outcome, potentially incorporates the other procedural risk variables, and is known to the perioperative physician prior to surgery. We generated receiver-operating characteristic (ROC) curves to quantify the discriminatory power of multivariable models, and used the DeLong test to compare the area under ROC curves. In all analyses, we considered a p-value (two-tailed) $<0.05$ to represent statistical significance. We used Stata version 15 (StataCorp LLC, College Station, TX, USA) for all analyses.

\section{Risk assessment tool development}

We used the multivariable model including the three-parameter noninvasive "low-risk focused" score to develop a risk assessment tool (PH perioperative risk or PHPR score) that can be calculated before surgery. We used this model to develop the PHPR score because of its superior performance characteristics and because additional invasive procedures are not required. The PHPR score (range 0-7) is calculated by adding a patient-level component and a procedural component. The patient-level component is the number of features in the composite score that do not reach "low-risk" thresholds (0-3). The procedural component is binary. "Low" risk procedures are assigned a value of 0 (no added risk). "Elevated" risk procedures are assigned a value of 4 , derived from the ratio of odds ratios for "elevated" procedural risk and number of composite score features that do not reach "low-risk" thresholds in the multivariable regression model $\left(e^{2.7} / e^{1.3}=e^{1.4}=4\right)$. For example, a patient with WHO FC II symptoms, NT-proBNP $=600 \mathrm{ng} \cdot \mathrm{L}^{-1}$, and $6 \mathrm{MWD}=250 \mathrm{~m}$ undergoing an endoscopy ("low" risk) would have a PHPR score of $(2+0)=2$, while the same patient undergoing a total knee replacement ("elevated” risk) would have a PHPR score of $(2+4)=6$. We used the distribution of PHPR scores to assign three risk categories ( $0-2=$ low, $3=$ intermediate, 4-7=high). Thresholds were intentionally set to include all patients having an "elevated" procedural risk procedure in the highest category.

\section{Results}

Study population, surgical procedures, and preoperative evaluation

During the study period, we prospectively identified 150 subjects undergoing noncardiac surgery (table 1). The cohort was predominantly female (76\%) with an average age of 59.7 years. Approximately half (52\%) had PAH, and $47.4 \%$ of these had connective tissue disease-associated PAH (supplemental table S2). Most subjects were receiving PAH-specific therapy at the time of surgery (72\%), and nearly all were deemed ASA classification of 3 or higher.

Most procedures (82\%) were performed at the Johns Hopkins Hospital, and $73.3 \%$ were classified as "low" risk using ACC/AHA guidelines [23]. Of 110 procedures classified as "low” risk, 54 (49\%) were endoscopies, primarily of the gastrointestinal tract (supplemental table S3). Endoscopic procedures are routinely staffed by cardiac anaesthesiology providers for $\mathrm{PH}$ patients at our institution [27]. Forty procedures (26.7\%) classified as “elevated" risk included primarily intraperitoneal, orthopaedic, or intrathoracic surgeries. $6 \%$ of all procedures were emergency procedures, 32\% were performed during inpatient hospitalisation, and 28\% lasted longer than $3 \mathrm{~h}$.

Outpatient clinical assessment by a PH specialist (67.3\%) or anaesthesiologist (21.3\%) was completed within 90 days of surgery for most patients. Among procedures performed at the Johns Hopkins Hospital, $75.6 \%$ were referred to the cardiac anaesthesiology liaison for case review. Most patients had WHO FC assessment (63\%), natriuretic peptide (NT-proBNP or BNP) measurement (55\%), and echocardiography (53\%) within 3 months before surgery, while fewer had 6MWD testing (23\%) or right heart catheterisation (21\%) in this interval (fig 1). Average values for the closest measurement before surgery are shown in supplemental table S4.

\section{Surgical outcomes}

The primary outcome was observed in 19 patients (12.7\%; table 2), including 7 deaths (4.7\%). Median time from surgery until death was 13 days (interquartile range (IQR) 5-23 days), with causes listed in supplemental table S5. Seventeen patients (11.3\%) developed serious postoperative complications. Among these, haemodynamic instability and respiratory failure were most common. Median hospital LOS was 1 day (IQR 0-6 days). Of the 37 patients (24.7\%) admitted to the ICU after surgery, 20 were planned 
TABLE 1 Preoperative variables

n $(\%)$ or mean \pm SD

\begin{tabular}{|c|c|}
\hline \multicolumn{2}{|l|}{ Patient characteristics } \\
\hline Age, years & $59.7 \pm 14.7$ \\
\hline Female & $114(76)$ \\
\hline Systemic sclerosis/mixed connective tissue disease & $54(36)$ \\
\hline \multicolumn{2}{|l|}{ WSPH group } \\
\hline Group 1 & $78(52.0)$ \\
\hline Group 2 & $23(15.3)$ \\
\hline Group 3 & $22(14.7)$ \\
\hline Group 4 & $15(10.0)$ \\
\hline Group 5 & $12(8.0)$ \\
\hline PAH-specific therapy & $108(72.0)$ \\
\hline \multicolumn{2}{|l|}{ American Society of Anesthesiologists class } \\
\hline 1 & $0(0.0)$ \\
\hline 2 & $4(2.7)$ \\
\hline 3 & $78(52.0)$ \\
\hline 4 & $67(44.7)$ \\
\hline 5 & $1(0.7)$ \\
\hline \multicolumn{2}{|l|}{ Procedural characteristics } \\
\hline \multicolumn{2}{|l|}{ ACC/AHA risk } \\
\hline Low & $110(73.3)$ \\
\hline Elevated & $40(26.7)$ \\
\hline Procedure at $\mathrm{JHH}$ & $123(82.0)$ \\
\hline Inpatient & $48(32.0)$ \\
\hline Emergency & $9(6.0)$ \\
\hline \multicolumn{2}{|l|}{ Duration $>3 \mathrm{~h}$} \\
\hline Yes & $42(28)$ \\
\hline No & $106(70.7)$ \\
\hline Unknown & $2(1.3)$ \\
\hline \multicolumn{2}{|l|}{ Preoperative assessments within 3 months of procedure } \\
\hline PH clinic visit & $101(67.3)$ \\
\hline Anaesthesia clinic visit & $32(21.3)$ \\
\hline \multicolumn{2}{|l|}{ Cardiac anaesthesia review ${ }^{\#}$} \\
\hline Yes & $93(75.6)$ \\
\hline No & $27(22.0)$ \\
\hline Unknown & $3(2.4)$ \\
\hline WHO FC assessment & $94(62.7)$ \\
\hline BNP/pro-BNP & $82(54.7)$ \\
\hline Echocardiography & $79(52.7)$ \\
\hline 6MWT & $34(22.7)$ \\
\hline $\mathrm{RHC}$ & $31(20.7)$ \\
\hline \multicolumn{2}{|c|}{$\begin{array}{l}\text { WSPH: World Symposium on Pulmonary Hypertension; PAH: pulmonary arterial hypertension; ACC/AH } \\
\text { American College of Cardiology/American Heart Association; JHH: Johns Hopkins Hospital; PH: pulmon } \\
\text { hypertension; WHO FC: World Health Organization functional class; BNP/pro-BNP: brain natriuretic pepti } \\
\text { pro-brain natriuretic peptide; 6MWT: 6-minute walk test; RHC: right heart catheterisation. \#: only amo } \\
\text { procedures performed at JHH. }\end{array}$} \\
\hline
\end{tabular}

(enhanced monitoring) and 17 were unplanned. ICU LOS was longer for unplanned admissions (median 4 versus 1 day; $\mathrm{p}<0.001$, supplemental figure $\mathrm{S} 1)$. Nineteen patients $(12.7 \%)$ were readmitted to the hospital within 30 days of surgery for clinical deterioration (supplemental table S6).

Associations between individual risk variables and surgical outcomes

Associations between individual preoperative variables and the primary outcome are shown in table 3 . Among patient-level variables, we observed no associations between patient age, sex, presence of systemic sclerosis, WSPH group, or use of PAH-specific therapy and the primary outcome. Patients with the highest ASA classification (4 or 5) had increased risk compared to those with ASA classification 2 or 3 . We did not find associations between preoperative evaluation by a $\mathrm{PH}$ specialist, anaesthesiologist, or case review by the cardiac anaesthesiology liaison and the primary outcome. WHO FC was associated with the primary outcome; subjects with WHO FC 3 or 4 limitations had greater risk than those with WHO FC 1 or 2 


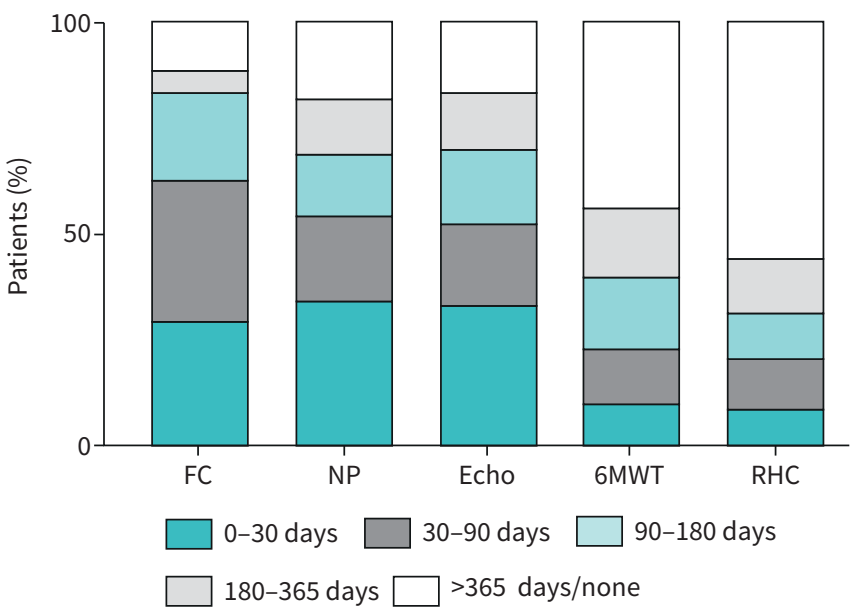

FIGURE 1 Percentage of patients having preoperative assessments during specified time intervals before surgery. FC: World Health Organization functional class; NP: natriuretic peptide; Echo: echocardiography; 6MWT: 6-min walk test; RHC: right heart catheterisation.

limitations (OR 5.87; $\mathrm{p}=0.02$ ). Natriuretic peptide levels were also associated with the primary outcome; subjects with NT-proBNP $>1400 \mathrm{ng} \cdot \mathrm{L}^{-1}$ (or BNP $>300 \mathrm{ng} \cdot \mathrm{L}^{-1}$ ) had higher odds of adverse events compared with those with NT-proBNP $<300 \mathrm{ng} \cdot \mathrm{L}^{-1}$ (or $\mathrm{BNP}<50 \mathrm{ng} \cdot \mathrm{L}^{-1}$ ) (OR 7.08; $\mathrm{p}=0.01$ ). We did not find associations between right atrial dilation, TAPSE, or pericardial effusion and the primary outcome. However, severe right ventricular (RV) dilation (OR 6.27; $\mathrm{p}=0.01$ ) and maximum Doppler tricuspid regurgitant velocity (OR 1.5 for every increase of $0.5 \mathrm{~m} \cdot \mathrm{s}^{-1}$; $\mathrm{p}=0.02$ ) were associated with increased risk. We found no associations between 6MWD or invasive haemodynamic measurements and the primary outcome.

All procedural variables examined were associated with the primary outcome. The strongest association was observed with procedures designated as "elevated" risk for major cardiovascular events, with an OR of 8.35 $(\mathrm{p}<0.001)$ compared to "low" risk procedures. Emergency procedures, those lasting $>3 \mathrm{~h}$, and procedures performed on inpatients were also associated with increased odds of the primary outcome (table 3).

Associations between composite PAH risk assessment scores and surgical outcomes

We used three approaches to generate composite PAH risk assessment scores, then used univariable logistic regression to estimate associations with the primary outcome. Only the three-parameter

TABLE 2 Outcomes

n (\%) or median (IQR)

\begin{tabular}{|lc|}
\hline Primary outcome & $19(12.7)$ \\
\hline Death within 30 days & $7(4.7)$ \\
\hline Serious postoperative complication & $17(11.3)$ \\
\hline Haemodynamic instability & $14(9.3)$ \\
\hline Respiratory failure & $10(6.7)$ \\
\hline Initiation of inhaled vasodilators & $4(2.7)$ \\
\hline Acute coronary syndrome & $0(0.0)$ \\
\hline Cerebrovascular accident & $1(0.7)$ \\
\hline Arrhythmia & $5(3.3)$ \\
\hline Renal failure & $3(2.0)$ \\
\hline Hepatic injury & $1(0.7)$ \\
\hline Sepsis & $2(1.3)$ \\
\hline Secondary outcomes & $1(0-6)$ \\
\hline Hospital LOS, days & $3(1-5)$ \\
\hline Intensive care unit LOS, days & $19(12.7)$ \\
\hline Hospital readmission within 30 days & \\
\hline IQR: interquartile range; LOS: length of stay. & \\
\hline
\end{tabular}


TABLE 3 Associations between preoperative variables and the primary outcome

\begin{tabular}{|c|c|c|c|}
\hline & \multicolumn{3}{|c|}{ Primary outcome reached } \\
\hline & n (\%) & OR $(95 \% \mathrm{CI})$ & $\mathrm{p}$-value \\
\hline \multicolumn{4}{|l|}{ Patient characteristics } \\
\hline Age, per year & & $1.01(0.97-1.04)$ & 0.76 \\
\hline \multicolumn{4}{|l|}{ Sex } \\
\hline Male & $7(19.4)$ & Reference & 0.17 \\
\hline Female & $12(10.5)$ & $0.49(0.18-1.35)$ & \\
\hline \multicolumn{4}{|c|}{ Systemic sclerosis/mixed connective tissue disease } \\
\hline No & $13(13.5)$ & Reference & 0.67 \\
\hline Yes & $6(11.1)$ & $0.80(0.28-2.24)$ & \\
\hline \multicolumn{4}{|l|}{ WSPH group } \\
\hline 1 & $9(11.5)$ & Reference & \\
\hline 2 & $2(8.7)$ & $0.73(0.15-3.65)$ & 0.70 \\
\hline 3 & $3(13.6)$ & $1.21(0.30-4.92)$ & 0.79 \\
\hline 4 & $3(20.0)$ & $1.92(0.45-8.12)$ & 0.38 \\
\hline 5 & $2(16.7)$ & $1.53(0.29-8.14)$ & 0.62 \\
\hline \multicolumn{4}{|l|}{ PAH-specific therapy } \\
\hline No & $6(14.3)$ & Reference & 0.71 \\
\hline Yes & $13(12.0)$ & $0.82(0.29-2.32)$ & \\
\hline \multicolumn{4}{|c|}{ American Society of Anesthesiologists risk } \\
\hline Low $(1-3)$ & $5(6.1)$ & Reference & 0.01 \\
\hline High (4-5) & $14(20.6)$ & $3.99(1.36-11.74)$ & \\
\hline \multicolumn{4}{|l|}{ Procedural characteristics } \\
\hline \multicolumn{4}{|c|}{ ACC/AHA procedure risk } \\
\hline Low & $6(5.5)$ & Reference & $<0.001$ \\
\hline Elevated & $13(32.5)$ & $8.35(2.90-23.99)$ & \\
\hline \multicolumn{4}{|l|}{ Emergent procedure } \\
\hline No & $15(10.6)$ & Reference & 0.01 \\
\hline Yes & $4(44.4)$ & $6.72(1.62-27.79)$ & \\
\hline \multicolumn{4}{|l|}{ Procedure $>3 \mathrm{~h}(\mathrm{~N}=148)$} \\
\hline No & $6(5.7)$ & Reference & $<0.001$ \\
\hline Yes & $13(31.0)$ & $7.47(2.61-21.39)$ & \\
\hline \multicolumn{4}{|l|}{ Inpatient } \\
\hline No & $8(7.8)$ & Reference & 0.01 \\
\hline Yes & $11(22.9)$ & $3.50(1.30-9.37)$ & \\
\hline \multicolumn{4}{|l|}{ Preoperative assessment } \\
\hline \multicolumn{4}{|l|}{ PH clinic within 90 days } \\
\hline No & $6(12.2)$ & Reference & 0.91 \\
\hline Yes & $13(12.9)$ & $1.06(0.38-2.98)$ & \\
\hline \multicolumn{4}{|c|}{ Anaesthesia clinic within 90 days } \\
\hline No & $16(13.6)$ & Reference & 0.53 \\
\hline Yes & $3(9.4)$ & $0.66(0.18-2.42)$ & \\
\hline \multicolumn{4}{|c|}{ Cardiac anaesthesia referral $(n=137)^{\#}$} \\
\hline No & $1(2.6)$ & Reference & 0.06 \\
\hline Yes & $16(16.3)$ & $7.41(0.95-57.98)$ & \\
\hline \multicolumn{4}{|l|}{ WHO FC $(n=141)$} \\
\hline Low $(\mathrm{I}, \mathrm{II})$ & $2(3.4)$ & Reference & 0.02 \\
\hline High (III, IV) & $14(17.1)$ & $5.87(1.28-26.90)$ & \\
\hline NT-proBNP (BNP), ng & & & \\
\hline$<300(<50)$ & $2(4.2)$ & Reference & \\
\hline $300-1400(50-300)$ & $4(8.3)$ & $2.09(0.36-12.00)$ & 0.41 \\
\hline$>1400(>300)$ & $12(23.5)$ & $7.08(1.49-33.56)$ & 0.01 \\
\hline Echocardiography & & & \\
\hline RA dilation $(n=146)$ & & & \\
\hline No & $2(4.2)$ & Reference & 0.07 \\
\hline Yes & $15(15.3)$ & $4.16(0.91-18.98)$ & \\
\hline RV dilation $(\mathrm{n}=147)$ & & & \\
\hline None & $3(6.0)$ & Reference & \\
\hline Mild & $5(11.1)$ & $1.96(0.44-8.71)$ & 0.38 \\
\hline Moderate & $2(8.3)$ & $1.42(0.22-9.14)$ & 0.71 \\
\hline
\end{tabular}




\begin{tabular}{|c|c|c|c|}
\hline & \multicolumn{3}{|c|}{ Primary outcome reached } \\
\hline & n (\%) & OR $(95 \% \mathrm{Cl})$ & $\mathrm{p}$-value \\
\hline Severe & $8(28.6)$ & $6.27(1.51-26.09)$ & 0.01 \\
\hline \multicolumn{4}{|l|}{ TAPSE, cm $(n=74)$} \\
\hline$\geqslant 1.8$ & $5(11.4)$ & Reference & \\
\hline$<1.8$ & $6(20.0)$ & $1.95(0.54-7.09)$ & 0.31 \\
\hline \multicolumn{4}{|l|}{ Effusion ( $N=145)$} \\
\hline No & $11(11.2)$ & Reference & 0.79 \\
\hline Yes & $6(12.8)$ & $1.16(0.40-3.35)$ & \\
\hline Max TR velocity, per $0.5 \mathrm{~m} \cdot \mathrm{s}^{-1}(\mathrm{n}=120)$ & & $1.50(1.05-2.13)$ & 0.02 \\
\hline \multicolumn{4}{|l|}{ 6MWD, $m(n=131)$} \\
\hline$>440$ & $2(7.7)$ & Reference & \\
\hline $165-440$ & $10(10.6)$ & $1.43(0.29-6.97)$ & 0.66 \\
\hline$<165$ & $2(18.2)$ & $2.67(0.33-21.87)$ & 0.36 \\
\hline \multicolumn{4}{|l|}{$\mathrm{RHC}$} \\
\hline \multicolumn{4}{|l|}{ RAP, $m m H g(n=144)$} \\
\hline$<8$ & $9(12.7)$ & Reference & \\
\hline $8.0-14$ & $8(14.3)$ & $1.15(0.41-3.20)$ & 0.79 \\
\hline$>14$ & $2(11.8)$ & $0.92(0.18-4.70)$ & 0.92 \\
\hline \multicolumn{4}{|l|}{ Cardiac index, $L \cdot \mathrm{min}^{-1} \cdot \mathrm{m}^{-2}(\mathrm{n}=137)$} \\
\hline$\geqslant 2.5$ & $4(12.5)$ & Reference & \\
\hline $2-2.49$ & $6(17.1)$ & $1.60(0.51 ; 5.05)$ & 0.42 \\
\hline$<2$ & $8(11.4)$ & $1.11(0.31 ; 3.98)$ & 0.88 \\
\hline \multicolumn{4}{|l|}{$S_{\mathrm{VO} 2}, \%(n=137)$} \\
\hline$>65$ & $7(9.5)$ & Reference & \\
\hline $60-65$ & $6(17.1)$ & $1.98(0.61 ; 6.41)$ & 0.25 \\
\hline$<60$ & $4(14.3)$ & $1.60(0.43 ; 5.94)$ & 0.49 \\
\hline \multicolumn{4}{|c|}{$\begin{array}{l}\text { WSPH: World Symposium on Pulmonary Hypertension; ACC/AHA: American College of Cardiology/American } \\
\text { Heart Association; WHO FC: World Health Organization functional class; NT pro-BNP/BNP: N-terminal } \\
\text { pro-hormone brain natriuretic peptide/pro-brain natriuretic peptide; RA: right atrial; RAP: right atrial pressure; } \\
\text { RV: right ventricular; TAPSE: tricuspid annular plane systolic excursion; TR: tricuspid regurgitant; 6MWD: } \\
\text { 6-minute walk distance; RHC: right heart catheterisation; } \mathrm{S}_{\mathrm{VO} 2} \text { : mixed venous oxygen saturation. \#: only among } \\
\text { procedures performed at Johns Hopkins Hospital or Johns Hopkins Bayview Medical Center. }\end{array}$} \\
\hline
\end{tabular}

noninvasive "low-risk focused" score was significantly associated with the primary outcome (OR 0.4, $\mathrm{p}=0.03$ ) (table 4). When the models were adjusted for procedural risk, all three composite PAH risk assessment scores were significantly associated with the primary outcome. In multivariable models, the strongest association was with the three-parameter noninvasive "low-risk focused" score where each additional "low-risk" feature was associated with a 70\% reduction in the estimated odds of an adverse surgical outcome $(p=0.01)$. We generated ROC curves to assess the discriminatory power of the multivariable models. Each had robust discriminatory power for the primary outcome, with a c-statistic at or above 0.8 (supplemental figure S2). However, when these multivariable ROC curves were compared to ROC curves for their component patient-level and procedural risk scores, only the three-parameter noninvasive "low-risk focused"-based multivariable model had better discrimination than both univariable models (figure 2 and supplemental figure S3). Finally, we used regression analyses to evaluate associations

TABLE 4 Association between composite patient-level risk scores and the primary outcome

\begin{tabular}{|c|c|c|c|c|}
\hline & \multicolumn{2}{|c|}{ Univariable } & \multicolumn{2}{|c|}{ Multivariable ${ }^{\#}$} \\
\hline & OR $(95 \% \mathrm{Cl})$ & p-value & OR $(95 \% \mathrm{Cl})$ & p-value \\
\hline Noninvasive low-risk focused & $0.4(0.2-0.9)$ & 0.03 & $0.3(0.1-0.8)$ & 0.01 \\
\hline Invasive and noninvasive low-risk focused & $0.7(0.4-1.2)$ & 0.15 & $0.5(0.3-1.0)$ & 0.05 \\
\hline Score and average & $1.9(0.8-4.3)$ & 0.15 & $3.6(1.2-10.6)$ & 0.02 \\
\hline
\end{tabular}




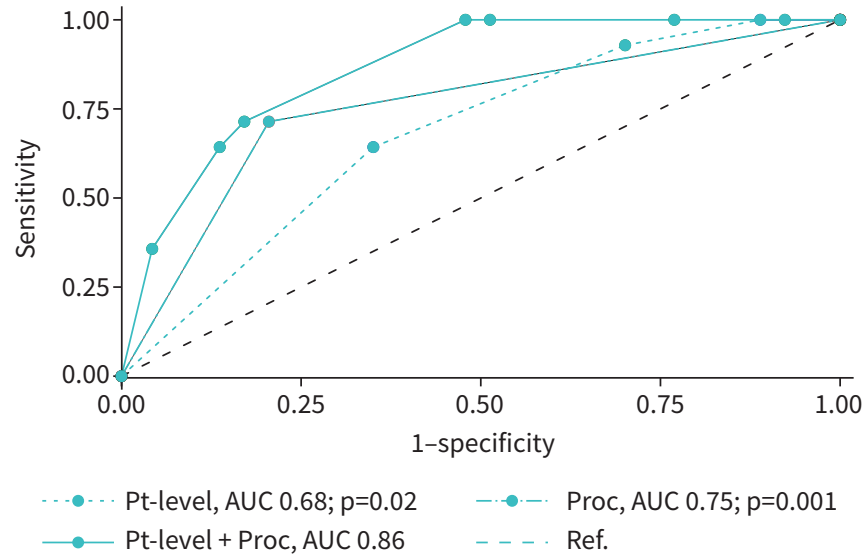

FIGURE 2 Receiver-operating characteristic (ROC) curves for logistic regression models including only patient-level (Pt-level) risk, only procedural (Proc) risk, or both (Pt-level+Proc). The area under the curve (AUC) for each model is shown in the legend. $p$-values are for DeLong test comparing ROC curves for each univariable model with the multivariable model.

between the composite PAH risk assessment scores and secondary outcomes (table 5). In univariable analyses, all approaches were associated with 30-day hospital readmission and none were associated with ICU LOS. The three-parameter noninvasive "low-risk focused" and "score and average" methods were also associated with hospital LOS in univariable models. After adjustment for procedural risk, all three composite PAH risk assessment scores were significantly associated with hospital LOS and odds of hospital readmission, while no score was associated with ICU LOS.

\section{Development of a perioperative PH risk assessment tool}

We used the three-parameter noninvasive "low-risk focused" multivariable model to develop a risk assessment tool (PHPR), as outlined in the methods. We used the distribution of patients to stratify PHPR scores into discrete categories that assign low $(0 \%)$, intermediate $(\leqslant 10 \%)$, or high $(>10 \%)$ perioperative risk (figure 3a). As anticipated, the percentage of subjects reaching the primary outcome increased steadily across PHPR categories $(\mathrm{p}<0.001)$. ROC curve analysis showed that the discriminatory power of the PHPR categories approximated that of the original multivariable model (figure $3 \mathrm{~b}$ ). As an exploratory analysis, we used ROC curves to compare the performance of the PHPR categories among patients with PAH

TABLE 5 Association between composite patient-level risk scores and secondary outcomes

\begin{tabular}{|c|c|c|c|c|}
\hline & \multicolumn{2}{|c|}{ Univariable } & \multicolumn{2}{|c|}{ Multivariable $^{\#}$} \\
\hline & $\boldsymbol{\beta}(95 \% \mathrm{Cl})^{4}$ & p-value & $\boldsymbol{\beta}(95 \% \mathrm{Cl})^{\natural}$ & $\mathrm{p}$-value \\
\hline \multicolumn{5}{|l|}{ Hospital LOS } \\
\hline Noninvasive low-risk focused & $-2.0(-3.5--0.5)$ & 0.01 & $-2.2(-3.6--0.8)$ & 0.003 \\
\hline Invasive and noninvasive low-risk focused & $-1.3(-2.7-0.1)$ & 0.06 & $-1.6(-2.9--0.3)$ & 0.02 \\
\hline Score and average & $3.4(0.7-6.0)$ & 0.01 & $4.3(1.9-6.8)$ & 0.001 \\
\hline \multicolumn{5}{|l|}{ Intensive care unit LOS } \\
\hline Noninvasive low-risk focused & $-1.0(-4.4-2.3)$ & 0.53 & $-1.8(-5.3-1.6)$ & 0.28 \\
\hline Invasive and noninvasive low-risk focused & $-0.4(-3.1-2.3)$ & 0.77 & $-0.7(-3.4-2.0)$ & 0.61 \\
\hline Score and average & $1.6(-2.2-5.4)$ & 0.40 & $2.6(-1.3-6.5)$ & 0.19 \\
\hline \multicolumn{5}{|l|}{ 30-day hospital readmission" } \\
\hline Noninvasive low-risk focused & $0.5(0.2-0.9)$ & 0.02 & $0.4(0.2-0.9)$ & 0.02 \\
\hline Invasive and noninvasive low-risk focused & $0.6(0.3-1.0)$ & 0.03 & $0.6(0.3-0.9)$ & 0.03 \\
\hline Score and average & $3.2(1.3-7.5)$ & 0.01 & $3.4(1.4-8.4)$ & 0.01 \\
\hline
\end{tabular}



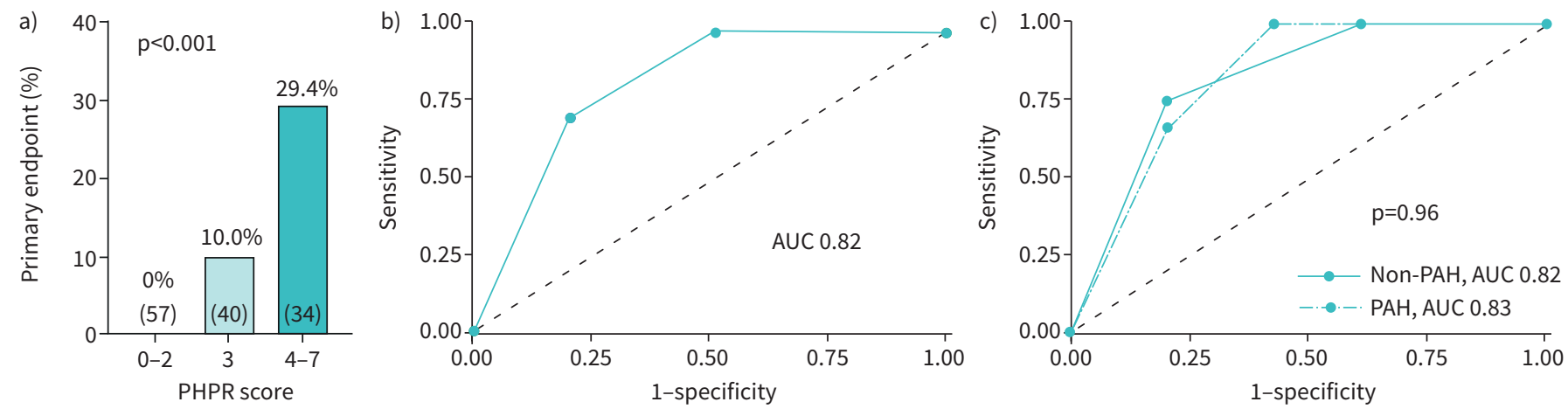

FIGURE 3 a) Percentage of patients reaching the primary end-point in each pulmonary hypertension perioperative risk (PHPR) category is shown. The number of patients in each category is shown in parenthesis. $p$-value is for Chi-squared test. b) Receiver-operating characteristic (ROC) curve for the PHPR categories. c) PHPR category ROC curves for patients with pulmonary arterial hypertension (PAH) and other forms of pulmonary hypertension are compared. The area under the curve (AUC) for each group is shown in the legend. $p$-value is for DeLong test comparing ROC curves.

(WSPH group I) and those without PAH (WSPH group II-V). We did not observe a difference in the discriminatory power of the PHPR score categories between the groups ( $\mathrm{p}=0.96$; figure $3 \mathrm{c})$.

\section{Discussion}

We describe a novel approach to perioperative risk assessment for $\mathrm{PH}$ patients undergoing noncardiac surgery. We showed that a multivariable model combining patient-level risk (composite PAH risk assessment scores) and procedural risk strongly predicts adverse surgical outcomes with improved discriminatory power compared to either approach alone. Importantly, all data required to evaluate risk is routinely collected in clinical practice, does not require additional invasive assessment, and is known to the provider before the procedure. We also provide "proof of principle" that the model can be converted to a clinically pragmatic risk score that may be useful for patients with all forms of $\mathrm{PH}$.

Several studies have described associations between individual patient-level variables and perioperative risk, but findings have been heterogeneous across studies and difficult to operationalise clinically. In a retrospective study of $145 \mathrm{PH}$ patients (excluding those with left heart disease) undergoing noncardiac procedures under general anaesthesia, RAMAKRISHNA et al. [1] showed that advanced WHO FC, RV hypertrophy, elevated RV systolic pressure relative to systemic systolic blood pressure and a history of pulmonary embolism were associated with perioperative morbidity and mortality. In another retrospective study of $196 \mathrm{PH}$ patients (also excluding those with left heart disease) undergoing noncardiac procedures, DeLJou et al. [5] showed that WHO FC and natriuretic peptide level are associated with postoperative complications. A prospective multicentre study conducted on 114 patients with PAH undergoing noncardiac nonobstetric surgeries identified elevated RAP and low 6MWD $(<399 \mathrm{~m})$ as patient-level factors associated with adverse perioperative outcome [3]. In a retrospective analysis of 173 patients undergoing right heart catheterisation and noncardiac surgery (96 with $\mathrm{PH}$ ), mean pulmonary artery pressure, ASA classification, and chronic renal insufficiency were identified as independent risk factors for postoperative morbidity [8]. We confirmed some of these relationships in our cohort (ASA classification, WHO FC, natriuretic peptide), but did not observe statistically significant associations for others. We also identified novel associations with two echocardiographic parameters (severe RV dilation, maximum Doppler tricuspid regurgitant velocity). This variability likely reflects the small sample sizes and limited statistical power for all of these studies, an inherent limitation to analyses of subjects with a rare disease who are often advised to avoid surgery $[10,11]$.

To address these limitations, we hypothesised that a composite "score" of variables routinely obtained in clinical practice would capture patient-level risk. Initially developed to assess prognosis in PAH patients [12-16], this approach combines clinical, functional, exercise, RV function and haemodynamic parameters to stratify patients into low, intermediate and high risk for 1-year mortality. Several scoring methods have been validated in estimating 1-year survival at the time of diagnosis [13], after treatment for 1 year [12, 14], and in several PAH subgroups $[17,18]$. More recently, these composite scores have been associated with health-related quality of life and rate of hospitalisations in PAH patients [19]. Our findings now extend this approach to perioperative risk assessment. In this domain, we found the strongest associations with the three-component noninvasive "low-risk focused" composite risk assessment score. However, we may have 
lacked sufficient power for statistical significance in univariable models with the other approaches, as there appeared to be meaningful trends in associations with the primary outcome and all approaches showed concordant associations with secondary outcomes. It is also possible that the three-component noninvasive model had superior performance in our analysis because the other approaches include invasive haemodynamic parameters, which were often obtained more than 1 year prior to surgery in our cohort (not reflective of perioperative clinical status). We believe this reflects clinical practice, as there are currently no strong recommendations for preoperative haemodynamic testing in patients with PAH.

Procedural factors intuitively inform perioperative risk, and procedural variables previously associated with adverse surgical outcomes among PH patients include inherent risk of major adverse cardiovascular event $[1,2,5]$, emergency surgery [2-4, 7], and procedures lasting longer than $3 \mathrm{~h}[2,24]$. We confirmed these relationships in our cohort, with the inherent procedural risk (elevated versus low) having the strongest association with the primary outcome. As this variable potentially incorporates other procedural risk factors (e.g. procedure length) and is known to the perioperative physician before the procedure, we elected to use it to adjust for procedural risk in multivariable models. This adjustment improved the discriminatory power of all three composite PAH risk assessment scores, but was significantly better than procedural risk alone at predicting adverse outcomes only when combined with the three-component noninvasive "low-risk focused" score. Although we lacked sufficient statistical power to determine whether procedural risk is an effect modifier or a confounder of the patient-level risk, we note that four of the seven deaths in our cohort occurred among patients with low procedural risk and high patient-level risk.

Clinically pragmatic risk assessment tools should aid providers in perioperative decision-making. We used the three-parameter noninvasive "low-risk focused" multivariable model to develop PHPR scores and stratify patients into risk categories (low, intermediate, high). This approach requires only clinical data routinely collected at regular intervals [11] and knowledge of the type of upcoming surgery. It avoids the need for repeat invasive haemodynamic assessment, which could delay surgery or generate additional risk. Furthermore, previous analyses have suggested that the noninvasive "low-risk focused" approach may be more accurate than the "score and average" approach for identifying patients with the lowest risk (highest long-term survival) [28]. As devised, we believe the PHPR score is most useful in identifying patients at the lowest perioperative risk that may be able to avoid additional workup or substantial adjustments to the perioperative plan. Because category thresholds intentionally restrict "elevated" risk procedures to the "high” PHPR category, the PHPR score incorporates patient-level risk to stratify patients undergoing "low" risk procedures as low or intermediate risk. Notably, a low PHPR score differentiated the $0 \%$ of patients who reached the primary outcome from the $10 \%$ of patients with an intermediate PHPR score who reached the primary outcome. Future studies in additional cohorts will be useful for further calibration of the PHPR score, potentially allowing additional discrimination between intermediate and high-risk patients.

\section{Limitations}

The cohort is limited to a single PH referral centre and included mostly low-risk procedures, which may limit generalisability. Other factors that may limit generalisability include the enrichment of our cohort with connective tissue disease-associated $\mathrm{PH}$ patients and routine incorporation of cardiac anaesthesiology in perioperative management. However, our observed rates of perioperative mortality and morbidity $(4.7 \%$ and $11.3 \%$ ) are consistent with previous reports (mortality $1-9.7 \%$ [1-8]; morbidity $6.1-42 \%$ [1, 3, 5]). The small sample size limited some analyses (as outlined above), but is also consistent with prior studies $[1-3,5,7,8]$. There was heterogeneity among subjects in the time between some preoperative patient-level variables and surgery, which could lead to ascertainment bias. This heterogeneity reflects the observational nature of our study and highlights a key knowledge gap in the field, namely the lack of evidence-based recommendations for specific preoperative testing protocols. Future prospective trials could evaluate the predictive power of a defined preoperative testing regimen (e.g. WHO FC, 6MWD, and NT-proBNP within 3 months of surgery). We were unable to assess the relationship between the US Registry to Evaluate Early and Long-term PAH Disease Management (REVEAL) 2.0 risk score, another composite PAH risk assessment tool $[15,16]$, and perioperative outcomes given the limited number of patients having sufficient information to calculate REVEAL 2.0 scores. Finally, we recognise that the PHPR score will require additional prospective validation, but present it here as "proof-of-principle" that a clinically pragmatic scoring system can be used to inform perioperative risk.

\section{Conclusion}

For PH patients undergoing noncardiac surgery, a composite PAH risk assessment score can be combined with procedural risk to improve perioperative risk assessment. When converted to a PHPR score, this approach provides a simple and clinically pragmatic tool for perioperative risk assessment for patients with $\mathrm{PH}$. 
Acknowledgements: The authors would like to acknowledge David N. Hager (Division of Pulmonary and Critical Care Medicine, Johns Hopkins University, Baltimore, MD, USA) for his insightful comments during the design of this study.

Conflict of interest: H.J. Hassan has nothing to disclose. T. Housten has nothing to disclose. A. Balasubramanian has nothing to disclose. C.E. Simpson has nothing to disclose. R.L. Damico has nothing to disclose. S.C. Mathai reports personal fees from Actelion, Liquidia and United Therapeutics outside the submitted work, and is a member of the Rare Disease Advisory Panel at the Patient Centered Outcomes Research Institute (PCORI). P.M. Hassoun serves on a scientific advisory board for MSD outside the submitted work. J. Steppan has nothing to disclose. P.J. Leary reports personal fees from Bayer, research support as a site PI for United Therapeutics and Actelion, and research grants from the American Heart Association, Pulmonary Hypertension Association, Chest Foundation, and NIH/NHLBI. All are outside the submitted work. T.M. Kolb has nothing to disclose.

\section{References}

1 Ramakrishna G, Sprung J, Ravi BS, et al. Impact of pulmonary hypertension on the outcomes of noncardiac surgery: predictors of perioperative morbidity and mortality. J Am Coll Cardiol 2005; 45: 1691-1699.

2 Price LC, Montani D, Jaïs X, et al. Noncardiothoracic nonobstetric surgery in mild-to-moderate pulmonary hypertension. Eur Respir J 2010; 35: 1294-1302.

3 Meyer S, McLaughlin VV, Seyfarth HJ, et al. Outcomes of noncardiac, nonobstetric surgery in patients with PAH: an international prospective survey. Eur Respir J 2013; 41: 1302-1307.

4 Memtsoudis SG, Ma Y, Chiu YL, et al. Perioperative mortality in patients with pulmonary hypertension undergoing major joint replacement. Anesth Analg 2010; 111: 1110-1116.

5 Deljou A, Sabov M, Kane GC, et al. Outcomes after noncardiac surgery for patients with pulmonary hypertension: a historical cohort study. J Cardiothorac Vasc Anesth 2020; 34: 1506-1513.

6 Smilowitz NR, Armanious A, Bangalore S, et al. Cardiovascular outcomes of patients with pulmonary hypertension undergoing noncardiac surgery. Am J Cardiol 2019; 123: 1532-1537.

7 Lai HC, Lai HC, Wang KY, et al. Severe pulmonary hypertension complicates postoperative outcome of non-cardiac surgery. Br J Anaesth 2007; 99: 184-190.

8 Kaw R, Pasupuleti V, Deshpande A, et al. Pulmonary hypertension: an important predictor of outcomes in patients undergoing non-cardiac surgery. Respir Med 2011; 105: 619-624.

9 Humbert M, Sitbon O, Chaouat A, et al. Survival in patients with idiopathic, familial, and anorexigen-associated pulmonary arterial hypertension in the modern management era. Circulation 2010; 122: $156-163$.

10 Taichman DB, Ornelas J, Chung L, et al. Pharmacologic therapy for pulmonary arterial hypertension in adults: CHEST guideline and expert panel report. Chest 2014; 146: 449-475.

11 Galiè N, Humbert M, Vachiery JL, et al. 2015 ESC/ERS Guidelines for the diagnosis and treatment of pulmonary hypertension: The Joint Task Force for the Diagnosis and Treatment of Pulmonary Hypertension of the European Society of Cardiology (ESC) and the European Respiratory Society (ERS): Endorsed by: Association for European Paediatric and Congenital Cardiology (AEPC), International Society for Heart and Lung Transplantation (ISHLT). Eur Heart J 2016; 37: 67-119.

12 Kylhammar D, Kjellström B, Hjalmarsson C, et al. A comprehensive risk stratification at early follow-up determines prognosis in pulmonary arterial hypertension. Eur Heart J 2018; 39: 4175-4181.

13 Hoeper MM, Kramer T, Pan Z, et al. Mortality in pulmonary arterial hypertension: prediction by the 2015 European pulmonary hypertension guidelines risk stratification model. Eur Respir J 2017; 50: 1700740.

14 Boucly A, Weatherald J, Savale L, et al. Risk assessment, prognosis and guideline implementation in pulmonary arterial hypertension. Eur Respir J 2017; 50: 1700889.

15 Benza RL, Gomberg-Maitland M, Miller DP, et al. The REVEAL Registry risk score calculator in patients newly diagnosed with pulmonary arterial hypertension. Chest 2012; 141: 354-362.

16 Benza RL, Gomberg-Maitland M, Elliott CG, et al. Predicting survival in patients with pulmonary arterial hypertension: the REVEAL risk score calculator 2.0 and comparison with ESC/ERS-based risk assessment strategies. Chest 2019; 156: 323-337.

17 Mercurio V, Diab N, Peloquin G, et al. Risk assessment in scleroderma patients with newly diagnosed pulmonary arterial hypertension: application of the ESC/ERS risk prediction model. Eur Respir J 2018; 52: 1800497.

18 Fayed H, Ahmad M, Abdelkhalek R, et al. 4970Validation of ESC/ERS 2015 guidelines risk score in patients with scleroderma associated pulmonary arterial hypertension (SSc-PAH). Eur Heart J 2019; 40: Suppl. 1: ehz746.0029.

19 Min J, Badesch D, Chakinala M, et al. Prediction of health-related quality of life and hospitalization in pulmonary arterial hypertension: the Pulmonary Hypertension Association Registry (PHAR). Am J Respir Crit Care Med 2021; 203: 761-764. 

Coll Cardiol 2013; 62: Suppl. 25, D34-D41.

21 Galiè N, Channick RN, Frantz RP, et al. Risk stratification and medical therapy of pulmonary arterial hypertension. Eur Respir J 2019; 53: 1801889.

22 Forfia PR, Fisher MR, Mathai SC, et al. Tricuspid annular displacement predicts survival in pulmonary hypertension. Am J Respir Crit Care Med 2006; 174: 1034-1041.

23 Fleisher LA, Fleischmann KE, Auerbach AD, et al. 2014 ACC/AHA guideline on perioperative cardiovascular evaluation and management of patients undergoing noncardiac surgery: executive summary: a report of the American College of Cardiology/American Heart Association Task Force on Practice Guidelines. Circulation 2014; 130: 2215-2245.

24 Yang El. Perioperative management of patients with pulmonary hypertension for non-cardiac surgery. Curr Rheumatol Rep 2015; 17: 15.

25 Ahmed K, Zygourakis C, Kalb S, et al. Protocol for urgent and emergent cases at a large academic level 1 trauma center. Cureus 2019; 11: e3973.

26 Kim D, Jules-Elysee K, Turteltaub L, et al. Clinical outcomes in patients with pulmonary hypertension undergoing total hip arthroplasty. Hss J 2014; 10: 131-135.

27 Steppan J, Diaz-Rodriguez N, Barodka VM, et al. Focused review of perioperative care of patients with pulmonary hypertension and proposal of a perioperative pathway. Cureus 2018; 10: e2072.

28 Hoeper MM, Pittrow D, Opitz C, et al. Risk assessment in pulmonary arterial hypertension. Eur Respir J 2018; 51: 1702606 